\title{
Functionalized Multi-Walled Carbon Nanotubes for Nitrogen Sensor
}

\author{
S. H. Pisal ${ }^{1}$, N. S. Harale ${ }^{2}$, T. S. Bhat ${ }^{2}$, H. P. Deshmukh ${ }^{3}$, P. S. Patil ${ }^{* 2}$ \\ ${ }^{1}$ Department of Physics, S. M. Joshi College, Hadapsar, Pune-411028, India \\ ${ }^{2}$ Thin Film Materials Laboratory, Department of Physics, Shivaji University, Kolhapur-416004, India \\ ${ }^{3}$ Department of Physics, Y.M.college, Pune, India
}

\begin{abstract}
Multiwalled Carbon Nanotubes (MWCNTs) produced by the arc discharge method are chemically functionalized with acid mixture. The functionalization of MWCNTs was confirmed by simple characterization techniques. This revealed that carboxylic group introduced, without disrupting main structure of MWCNTs. The gas sensing performance of the functionalized MWCNTs towards $\mathrm{NO}_{2}$ is studied. The highest sensitivity of 26.88 $\%$ for 100 ppm of $\mathrm{NO}_{2}$ at $27^{\circ} \mathrm{C}$ is observed towards functionalized MWCNTs.
\end{abstract}

Keywords: Carbon nanotubes, Functionalization, Reflux, Response time, Carboxylation

\section{Introduction}

CNTs are sheets of carbon atoms arranged in hexagons that curl into a tube [1] possessing unique electrical and electronic properties. The extremely high surface-to-volume ratio and hollow structure of CNTs is ideal for the adsorption of gas molecules [2]. Many researchers [3-5] have shown that as produced CNTs has the tendency to exist in bundles rather than as individual tubes, because of strong Vander Waals interactions, leading to insolubility in most organic media, and therefore limiting the range of applications. A common technique to improve dispersion and achieve optimum utilization of CNTs is its chemical functionalization [6, 7]. The development of highly sensitive chemical sensors is an attractive area of research because of their widespread applications in the industry, agriculture, environment, biomedicine and pharmaceutics. The principles of CNT- gas sensors for the detection of different gases are based on changes in electrical properties induced by charge transfer with the gas molecules [8]. A study of a pristine CNT-based sensor reports slow and incomplete recovery $[9,10]$. To overcome these limitations, improvement of interfacial interaction can be achieved by the functionalization of CNTs. The polar groups on the nanotubes surface increase the adsorption affinity of the electron-donor or acceptor gases and enhance their sensing performance [8].

\section{Experimental}

$100 \mathrm{mg}$ pristine MWCNTs were refluxed with of $\mathrm{H}_{2} \mathrm{SO}_{4}+\mathrm{HNO}_{3}(3: 1)$ at $55^{\circ} \mathrm{C}$ for $11 \mathrm{~h}$. Then reaction mixture was stirred at $40{ }^{\circ} \mathrm{C}$ for $12 \mathrm{~h}$ and diluted three times with distilled water, filtered using centrifuge machine having $8,000 \mathrm{rpm}$. The process of centrifugation and washing off with distilled water repeated till neutral $\mathrm{pH}$. Then the sample dried in vacuum oven at $50^{\circ} \mathrm{C}$ for $24 \mathrm{~h}$ gives carboxylated MWCNTs (MWCNT$\mathrm{COOH}$. This leads to opening the caps of MWCNTs [11]. A certain amount of MWCNT-COOH powder was suspended in $20 \mathrm{ml}$ distilled water in beaker by ultrasonic stirring for $20 \mathrm{~min}$. After well dispersion in distilled water thin films made on glass substrate using dip coating method. Then this film was tested upon exposed to $\mathrm{NO}_{2}$ gas. The sensing properties of the film were studied at different temperatures and at different concentrations.

\section{Reaction Mechanism}

The possible reaction takes place during Carboxylation of CNTs is given in "equation 1". Fig.1 describes gas sensing mechanism of functionalized CNTs towards $\mathrm{NO}_{2}$ gas.

$$
\mathrm{MWCNT}+\mathrm{H}_{2} \mathrm{SO}_{4}: \mathrm{HNO}_{3} \rightarrow \mathrm{MWCNT}-\mathrm{COOH}+\mathrm{H}_{2} \mathrm{O}+\mathrm{SO}_{2}+2 \mathrm{NO}_{2}
$$

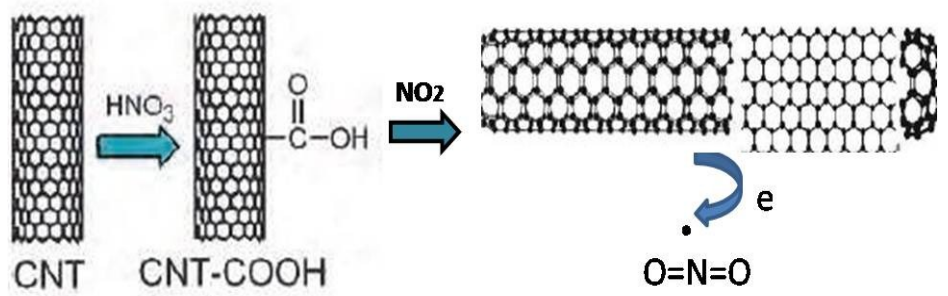

Fig.1: Reaction mechanism takes place during functionalization and sensing response 
X-Ray Diffraction (XRD) Studies

\section{Results and Discussion}

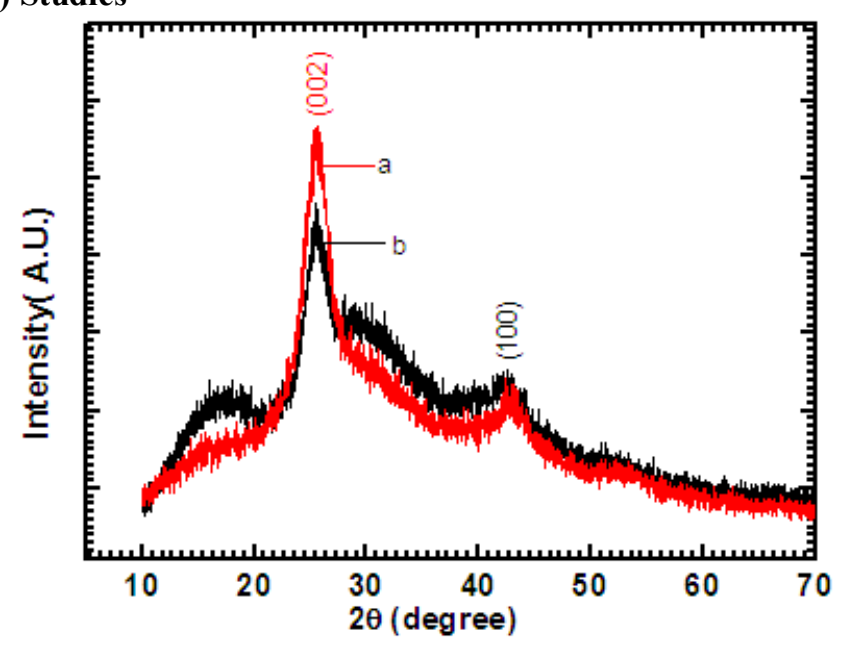

Fig.2: XRD spectra of the a) pristine MWCNT b) MWCNT-COOH

XRD patterns of the pristine MWCNT and MWCNTs-COOH, samples are shown in Fig. 2. The pristine MWCNT samples revealed the presence of two peaks at $25.70^{\circ}$ and $42.43^{\circ}$ corresponds to (002) and (100) planes of the carbon atoms respectively with interlayer spacing (34 nm) [12]. There is no drastic change in the position of characteristic peaks of pristine MWCNTs and MWCNT-COOH was observed, which suggests that MWCNTs are retained with their original structure after functionalization.

\section{Fourier Transform Infrared (FT-IR) Studies}

FT-IR spectra in the range $4000-400 \mathrm{~cm}^{-1}$ were recorded in order to investigate the nature of the chemical bonds formed. The FT-IR spectra of the pristine and functionalized MWCNTs are shown in Fig. 3

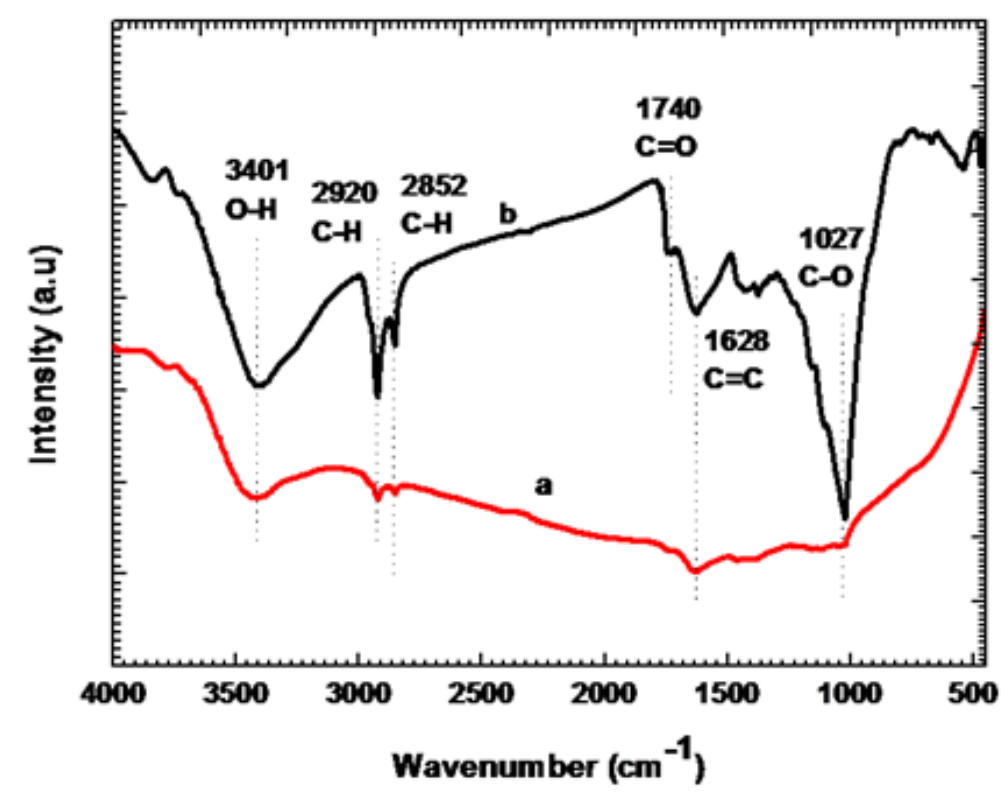

Fig. 3: FT-IR spectra of the (a) pristine MWCNTs and (b) MWCNT-COOH powder samples

Fig. 3(b) shows characteristic peaks of MWNT-COOH at $1027 \mathrm{~cm}^{-1}(\mathrm{C}-\mathrm{O}), 1628 \mathrm{~cm}^{-1}(\mathrm{C}=\mathrm{C}), 1740 \mathrm{~cm}^{-1}$ $(\mathrm{C}=\mathrm{O})$, and $3401 \mathrm{~cm}^{-1}(-\mathrm{OH})$. As compared with the FT-IR spectrum of pristine MWCNTs (Fig. 3 (a)), the peaks at $\simeq 1740$ and $1027 \mathrm{~cm}^{-1}$ in Fig. 3(b) were from the stretching vibration of $\mathrm{C}=\mathrm{O}$ and $-\mathrm{C}-\mathrm{O}$ groups in the carboxyl group (-COOH), respectively $[13,14]$.

\section{Scanning Electron Microscopy (SEM) Studies}

SEM is used to observe the morphologies of the MWCNTs. In Fig. 4(a) tubes of pristine MWCNTs can be clearly seen. Whereas the amorphous carbon layer is deposited on the surface of MWCNT-COOH ((Fig. 4 
(b)). Compared with pristine MWCNTs, the functionalized MWCNTs are shorter in length. The acid treatment can fragment the MWCNTs [15]. Many entangled clusters of functionalized MWCNTs are observed. Broken or damaged MWCNTs are more amenable to functionalization than pristine CNTs, due to the higher concentration of defects.
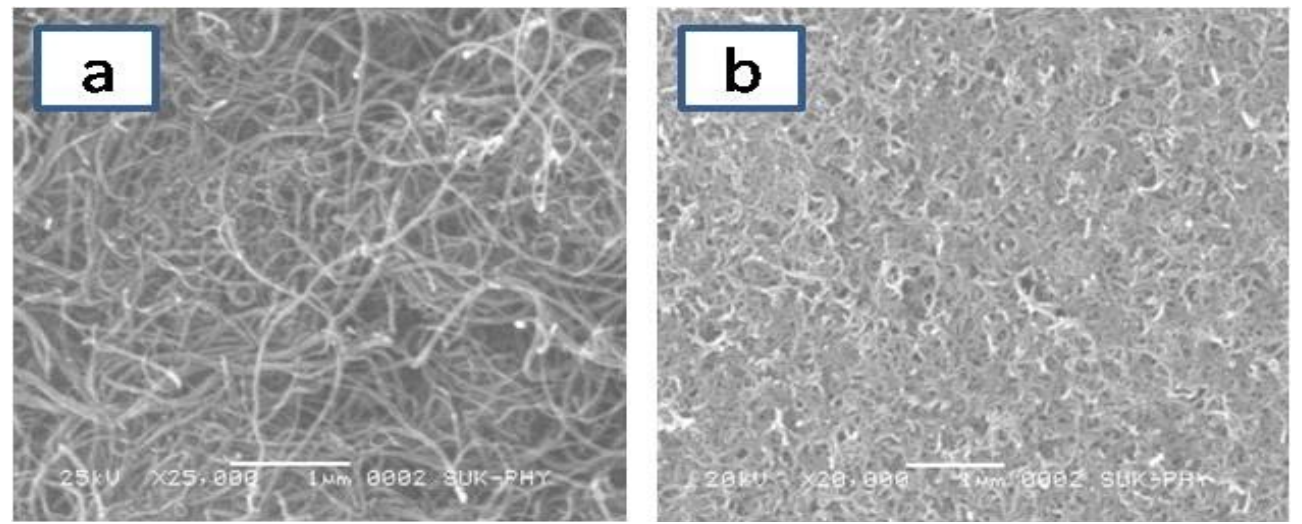

Fig. 4: SEM images of the (a) pristine MWCNT (b) MWCNT-COOH

\section{Gas sensing Study}

In present study, the sensing properties of MWCNT-COOH was studied at different temperatures from $50{ }^{\circ} \mathrm{C}$ to $225^{\circ} \mathrm{C}$ and at different concentrations $(50,100$ and $200 \mathrm{ppm})$ toward $\mathrm{NO}_{2}$ under continuous flow of $\mathrm{NO}_{2}$ gas. The response (S) of the sensor is expressed as the ratio of the change in resistance $(\Delta \mathrm{R})$ upon exposure to absorbed vapor to the resistance $\left(\mathrm{R}_{\mathrm{A}}\right)$ of the sample in the air [16] as shown by "equation 2".

$$
\mathrm{S}=\frac{\Delta \mathrm{R}}{\mathrm{R}_{\mathrm{A}}} \mathrm{X} 100 \%
$$

Where $\Delta \mathrm{R}$ is the resistance difference between $\mathrm{R}_{\mathrm{G}}$ and $\mathrm{R}_{\mathrm{A}}$, and $\mathrm{R}_{\mathrm{G}}$ denote the resistance of the sample measured in the presence of absorbed vapor. Fig. 5 demonstrates the dynamic response of MWCNT-COOH sensor on exposure to $\mathrm{NO}_{2}$ at different vapour concentration, viz., 50,100 and $200 \mathrm{ppm}$. Table1 summarizes response time, recovery time and sensitivity of MWCNT-COOH towards $\mathrm{NO}_{2}$ at different temperatures and concentrations.

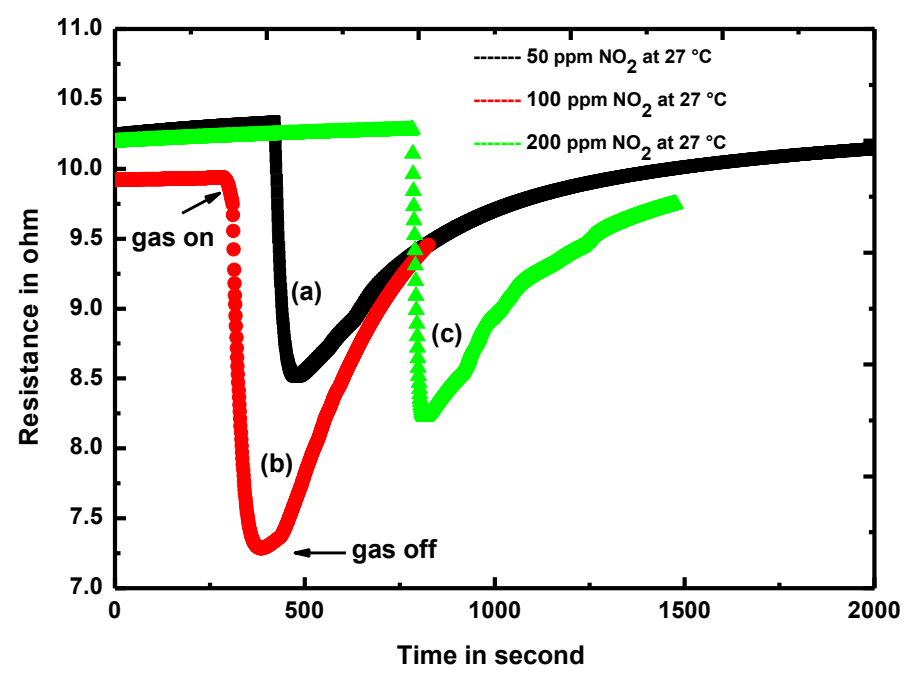

Fig. 5: Sensing response of the MWCNT-COOH at (a) $50 \mathrm{ppm}$ (b) $100 \mathrm{ppm}$ (c) $200 \mathrm{ppm}$ 
Table1. Sensing response of MWCNT-COOH towards $\mathrm{NO}_{2}$ at different temperatures

\begin{tabular}{|c|c|c|c|c|}
\hline Temperature $\left({ }^{\circ} \mathrm{C}\right)$ & $\begin{array}{c}\text { G as } \\
\text { concentration } \\
(\mathrm{ppm})\end{array}$ & $\begin{array}{c}\text { Response } \\
\text { time }(\mathrm{sec})\end{array}$ & $\begin{array}{c}\text { Recovery } \\
\text { Time (sec) }\end{array}$ & $\begin{array}{c}\text { Response } \\
(\%)\end{array}$ \\
\hline 50 & 50 & 565 & 158 & 13.69 \\
\hline 75 & 100 & 351 & 3 & 10.27 \\
\hline 100 & 100 & 464 & 1 & 9.06 \\
\hline 150 & 100 & 114 & 113 & 16.25 \\
\hline 200 & 100 & 54 & 18 & 11.62 \\
\hline 225 & 100 & 48 & 2 & 9.24 \\
\hline room & 50 & 265 & 265 & 17.98 \\
\hline room & 100 & $\mathbf{2 3 0}$ & $\mathbf{2 8 8}$ & $\mathbf{2 6 . 8 8}$ \\
\hline room & 200 & 240 & 251 & 20.25 \\
\hline
\end{tabular}

\section{Conclusion}

We reported here a synthesis of carboxylated MWCNTs using a chemical method. Treatment with strong acid mixture results in formation of carboxyl groups. The XRD spectra shows that the intensity of the (002) peaks decreases monotonically as MWCNTs gets functionalized. The FT-IR spectra confirm the presence of $-\mathrm{COOH}$, functional group. SEM image shows that acid-treatments shorten the length of MWNTs. MWCNT$\mathrm{COOH}$ exhibited good response towards $\mathrm{NO}_{2}$ at $27^{\circ} \mathrm{C}$ for $100 \mathrm{ppm}$.

\section{References}

[1]. M. Ge and K. Sattler, Scanning tunnelling microscopy of single-shell nanotubes of carbon. Appl Phys Lett; 65, 1994, 2284

[2]. Y. Wang, T.W. John and Yeow, A Review of Carbon Nanotubes-Based Gas Sensor, Sensors Volume, 10(1155), 2009,493904.

[3]. S. Iijima, Helical microtubules of graphitic carbon, Nature, 354,1991,56.

[4]. N. Tagmatarchis and M. Prato, Carbon-based materials: from fullerene nanostructures to functionalized carbon nanotubes, Pure and Applied Chemistry 77, 2005, 1675.

[5]. A. Hirsch, Functionalization of Single-Walled Carbon Nanotubes, Angew Chem Int Ed, 41, $2002,1853$.

[6]. S. Niyogi, H. Hu, M. A. Hamon et al., Chromatographic purification of soluble single-walled carbon nanotubes, Amer Chem Soc, 123, 2001,733

[7]. A. P. Yu, E. Bekyarova, M. E. Itkis et al., Application of centrifugation to the large-scale purification of electric arc-produced single-walled carbon nanotubes. Amer Chem Soc, 128, 2006, 9902.

[8]. D.W. Hatchett, M. Josowicz, Composites of intrinsically conducting polymers as sensing Nanomaterials, Chemical Reviews, 108(2),2008,746.

[9]. K. J. Franklin, N. R. Zhou, C. Chaplin,M. G. Peng, S. Cho, K. Dai, Nanotubes molecular wires as chemical sensors, Sci Mag, 287, 2000,622 .

[10]. W. Li, J. Lu , Y.Ye, Q. Cinke, M. Han, J. Meyyappan, Carbon nanotubes sensors for gas and organic vapor detection, Nano Lett, 79, 2003,929.

[11]. P. M. Kadam, N. L.Tarwal, S. S. Mali, H. P. Deshmukh and P. S. Patil, Enhanced electrochromic performance of f-MWCNT-WO3 composite. Electrochimica Acta, 58, 2011, 556

[12]. Y. Cheng, W. Li, X. Fang, J. Liu. W. Xu and C. Yan, Modified Multi-walled carbon nanotubes/ Ag nano partical composites catalyst for oxygen reduction reaction in alkaline solution, Electrochimica Acta, 11, 2013, 635.

[13]. C. Velasco, A. Martinez, V. M. Castano, Hydrogen bonding of polystyrene latex nano spheres to open-end cap and sidewall carbon nanotubes, Phys Chem B108, 2004, 18866.

[14]. S. G. Wang, R. Wang, P. J. Sellin, Q. Zhang, DNA biosensors based on self-assembled carbon nanotubes, Biochem Biophys Res Commun, 325(4), 2004, 1433.

[15]. P. M. Ajayan, T.W. Ebbesen, T. Ichihashi, S.Iijima, K. Tanigaki and H. Hiura, Opening carbon nanotubes with oxygen and implications for filling, Nature 362, 1993,522.

[16]. P. Kar, A. Choudhury, Carboxylic acid functionalized multi-walled carbon nanotubes doped polyaniline for chloroform sensors, Sensors and Actuators B, 183, 2013, 25. 